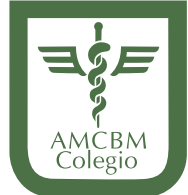

Revista Mexicana de

Cirugía Bucal y Maxilofacial

Vol. 17, Núm. 2

Mayo-Agosto 2021. pp. 55-61

Artículo original

\title{
Análisis de patrones de cierre velofaríngeo en pacientes con paladar hendido al realizar las técnicas quirúrgicas de esfinteroplastia lineal y doble z-plastia de Furlow. Estudio piloto
}

Analysis of velopharyngeal closure patterns in patients with cleft palate when performing the linear sphincteroplasty and Furlow double z-plasty surgical techniques. Pilot study

\author{
María José Hernández Álvarez,* Hernán Castilla Canseco, \\ Guadalupe Góngora Cadena, ${ }^{\S}$ Fabiola Salgado Chavarría ${ }^{\text {II }}$
}

\section{RESUMEN}

Objetivo: Correlacionar el tipo de patrón de cierre velofaríngeo en los pacientes con paladar hendido utilizando dos tipos diferentes de cierre quirúrgico con las técnicas de esfinteroplastia lineal de Bardach y doble z-plastia de Furlow. Material y métodos: Se realizó un estudio piloto transversal, retrospectivo, en el Hospital Pediátrico de Peralvillo, en el periodo de enero de 2017 a febrero de 2020. Resultados: Se registraron 118 pacientes con paladar hendido que fueron tratados quirúrgicamente, sólo 16 cumplieron con los criterios de inclusión, 11 fueron hombres $(68.75 \%)$ y cinco mujeres $(31.25 \%)$. La técni-

\section{ABSTRACT}

Objective: Correlate type of velopharyngeal closure pattern in patients with cleft palate using two different types of surgical closure with the Bardach linear sphincteroplasty and Furlow double z-plasty techniques. Material and methods: A retrospective, cross-sectional pilot study was realized at Peralvillo Pediatric Hospital, in the period from January 2017 to February 2020. Results: 118 patients with cleft palate who were treated surgically were registered, only 16 fulfilled the inclusion criteria, 11 were male (68.75\%), five female (31.25\%). The main surgical technique used was Bardach 62.5 and the double

* Residente de Cirugía Oral y Maxilofacial, Universidad Nacional Autónoma de México (UNAM), México.

¥ Jefe del Servicio de Cirugía Maxilofacial del Hospital Pediátrico de Peralvillo.

\& Otorrinolaringología y Cirugía de Cabeza y Cuello del Hospital Pediátrico de Peralvillo

" Responsable de Investigación de la Clínica de Cirugía Oral y Maxilofacial, UNAM.

Correspondencia

Dr. Hernán Castilla Canseco

Hospital Pediátrico de Peralvillo.

Calzada San Simón Núm. 14, Col. San Simón Tolnahuac, 06920, Alcaldía Cuauhtémoc, CDMX.

Tel: 55 3398-2851/72 2267-0189

E-mail:cmfhcc@gmaill.com

Citar como: Hernández ÁMJ, Castilla CH, Góngora CG, Salgado CF. Análisis de patrones de cierre velofaríngeo en pacientes con paladar hendido al realizar las técnicas quirúrgicas de esfinteroplastia lineal y doble z-plastia de Furlow. Estudio piloto. Rev Mex Cir Bucal Maxilofac. 2021;17 (2): 55-61. https://dx.doi.org/10.35366/102428 
ca quirúrgica principalmente utilizada fue Bardach 62.5 y la doble Z Furlow se utilizó en seis ocasiones (37.5\%). El patrón de cierre circular fue el más prevalente (media 10.1 años) realizada en ocho hombres y tres mujeres, seguido del coronal (media 5.2 años) ejecutada en cuatro niños, dos femeninos y dos masculinos y rodete de Passavant (media 14 años) efectuada en un masculino. Conclusiones: Pocos estudios describen los patrones de cierre velofaríngeo en pacientes con paladar hendido, pero concuerdan que fisiológicamente el patrón circular es el más común en el ámbito mundial. Este estudio puede ser utilizado para seguir realizando otras investigaciones en las cuales se tenga una mayor muestra para el análisis de patrones de cierre velofaríngeo, y comparen técnicas quirúrgicas para saber con cuál de ellas se obtiene el patrón de cierre velofaríngeo más adecuado para la fonación en pacientes de habla hispana.

Palabras clave: Paladar hendido, esfínter velofaríngeo, fonación, insuficiencia velofaríngea.

\section{INTRODUCCIÓN}

El paladar hendido es un desorden congénito común dentro de las hendiduras orofaciales de etiología multifactorial, ${ }^{1}$ ocurre embriológicamente en la sexta semana de vida intrauterina cuando los procesos maxilares sufren una falta de fusión, lo que provoca una comunicación de las cavidades nasal y bucal, alterando procesos fisiológicos como la respiración, fonación y masticación. ${ }^{2}$

El lenguaje humano es soportado por estructuras anatómicas complejas y procesos fisiológicos matizados. El mecanismo velofaríngeo funciona como esfínter que interviene en la fonación, deglución, respiración y la audición, el cual consta de una válvula muscular que se extiende de la parte ulterior del paladar duro a la pared faríngea posterior e incluye al velo (paladar blando), las paredes faríngeas laterales y la pared faríngea posterior. . $^{3,4,17}$

La función del mecanismo velofaríngeo es crear un sello hermético entre el velo y las paredes faríngeas para separar las cavidades oral y nasal para varios propósitos, incluyendo el lenguaje. 3,4,17

En pacientes con paladar hendido, la disfunción velofaríngea es una condición clínica compleja que se divide en dos grandes entidades: insuficiencia velofaríngea, que es definida como la inhabilidad para el completo cierre del esfínter velofaríngeo, es decir un defecto estructural (un velo del paladar corto); mientras que la incompetencia velofaríngea consiste en un movimiento insuficiente del velo del paladar, esto es, un defecto fisiológico (movimiento del velo
Z Furlow was used on six occasions (37.5\%). The circular closure pattern was the most prevalent [mean 10.1 years] performed in eight males and three females, followed by the coronal [mean 5.2 years] performed in four children, two females and two males, and Passavant's ridge [mean 14 years] performed in a masculine. Conclusions: Few studies describe velopharyngeal closure patterns in patients with cleft palate, but they agree that physiologically the circular pattern is the most common worldwide. This study can be used to continue carrying out other investigations in which there is a larger sample for analysis of velopharyngeal closure patterns and comparing surgical techniques to find out which of them is the most appropriate velopharyngeal closure pattern obtained for phonation in Spanish speaking patients.

Keywords: Cleft palate, velopharyngeal sphincter, phonation, velopharyngeal insufficiency.

del paladar insuficiente), siendo las principales consecuencias escape de aire nasal o hipernasalidad, soplido nasal audible, ronquido nasal, falta de nitidez en las consonantes y errores en la articulación de palabras, teniendo como resultado un lenguaje casi incomprensible. ${ }^{5,6,23}$

Los patrones de cierre velofaríngeo se clasifican según el grado en el que los componentes del esfínter velofaríngeo son activados durante la fonación, descritos inicialmente por Skolnick y Crofort (1973): coronal, sagital, circular y circular con rodete de Passavant; estos patrones de cierre se establecen dependiendo de los movimientos relativos del velo y de las paredes posterior y laterales de la faringe al cierre del esfínter (Figura 1).3,5,7-9,18

Los métodos más comunes para evaluar la función faríngea son subjetivos y objetivos: 1) evaluación perceptual del habla por un terapista de lenguaje con un criterio estandarizado; 2) examinación clínica por el cirujano maxilofacial; y 3) evaluación sistemática transoral y nasal con nasoendoscopia por un otorrinolaringólogo estandarizado. ${ }^{10}$

Diversas técnicas quirúrgicas son usadas para el tratamiento de la insuficiencia velofaríngea, las cuales tienen como objetivo común crear una obstrucción parcial permanente del espacio velofaríngeo. Las veloplastias son técnicas quirúrgicas que se realizan sobre el velo del paladar con el objetivo de alargarlo mediante el desplazamiento de los colgajos hacia atrás, o utilizando el principio de desplazamiento posterior (push-back); dentro de las cuales, la más utilizada es la doble z- plastia de Furlow. ${ }^{11,12,19}$ Por otro 
lado, las esfinteroplastias o faringoplastias dinámicas son técnicas quirúrgicas que actúan sólo a nivel de las paredes faríngeas (laterales y posteriores) con el objetivo de disminuir los diámetros de la orofaringe, a nivel de los surcos laterales rinofaríngeos, reconstruyendo un verdadero esfínter orofaríngeo de dimensiones reducidas, un ejemplo de éstas es la técnica de Bardach. 3,4,16,20

En la literatura mundial, existen muy pocos estudios que hablen acerca de los patrones velofaríngeos en pacientes con paladar hendido y menos técnicas quirúrgicas eficaces para la obtención de un adecuado patrón de cierre. El objetivo del presente artículo es correlacionar el tipo de patrón de cierre velofaríngeo en los pacientes con paladar hendido utilizando dos tipos diferentes de cierre quirúrgico con las técnicas de esfinteroplastia lineal de Bardach y doble z-plastia de Furlow.

\section{MATERIAL Y MÉTODOS}

Se trata de un estudio piloto transversal, retrospectivo. Se observaron 118 pacientes con paladar hendido que fueron tratados quirúrgicamente en el

Anatomía velofaríngea

Pared faríngea posterior

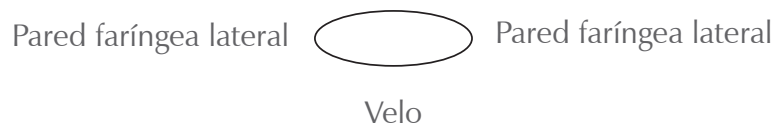

Patrones de cierre Dirección del movimiento

Coronal

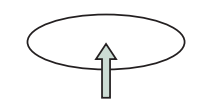

Sagital

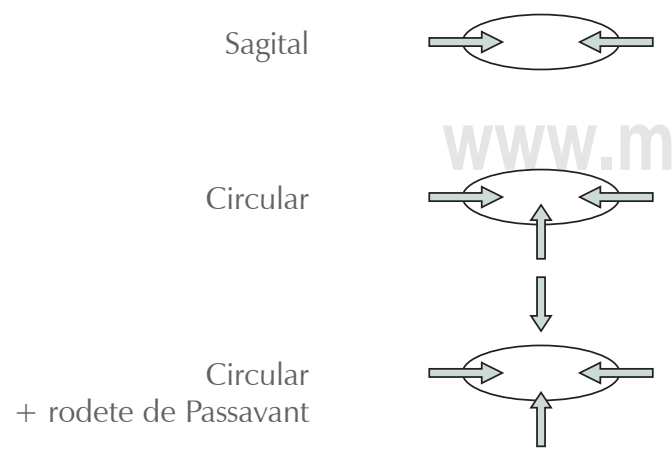

Figura 1: Patrones de cierre velofaríngeo. Tomado y modificado de: Skolnick $M$ et al. ${ }^{5}$
Hospital Pediátrico de Peralvillo, en el periodo de enero de 2017 a febrero de 2020, los criterios de inclusión fueron los siguientes: pacientes de ambos sexos, de tres a 20 años de edad, que presentan paladar hendido primario unilateral derecho 0 izquierdo, paladar submucoso, paladar bilateral que no presentaran enfermedad sistémica concomitante, que se sometieron a cirugía con las técnicas de esfinteroplastia lineal de Bardach y doble z-plastia de Furlow y contaran con consentimiento informado y asentimiento. Los criterios de exclusión: pacientes con paladar hendido secundario, o con veloplastias/ esfinteroplastia previas de paladar, pacientes sindrómicos o con enfermedades sistémicas. Los criterios de eliminación: pacientes a los cuales no se les realizaron las técnicas quirúrgicas de esfinteroplastia lineal de Bardach y doble z-plastia de Furlow y aquéllos que no contaron con la edad necesaria para realizar la nasofibroendoscopia.

Protocolo de tratamiento: las intervenciones quirúrgicas ocurrieron en el periodo de tiempo de enero de 2017 a febrero de 2020. Las dos técnicas que se utilizaron fueron la esfinteroplastia lineal de Bardach y la doble z-plastia de Furlow, las cuales fueron realizadas por el mismo cirujano maxilofacial. Los pacientes iniciaron su terapia de lenguaje al mes de postoperados.

La nasofibroendoscopia (NFE) se llevó acabo seis meses posteriores al acto quirúrgico. Este procedimiento fue realizado por la otorrinolaringóloga de dicho hospital usando un nasofibroendoscopio de fibra flexible Richard Wolf ${ }^{\circledast}$ de $3.5 \mathrm{~mm}$ de diámetro de longitud nL', $300 \mathrm{mcm}$ de flexión en la punta de trabajo (serie 7223.001), con canal de trabajo 1.1, ángulo de imagen $90^{\circ}$, una dirección de visión $0^{\circ}$ y un ángulo de curvatura de $130^{\circ}$ hacia proximal y distal, con una cabeza de cámara de 50 Hertz (serie5514961). La NFE se realizó de acuerdo a las recomendaciones de estandarización: el paciente se coloca sentado en un ángulo de $90^{\circ}$ con respecto al piso, se coloca una solución de lidocaína ${ }^{\circledR}$ simple a $1 \%$ y oximetazolina ${ }^{\circledR}$ a $0.025 \%$ en dilución $50 / 50$ con dispersor en las fosas nasales, tres disparos en cada fosa nasal dirigidos hacia la cabeza, cuerpo y cola del cornete inferior y se da una latencia de cinco minutos, realizando una rinoscopia antero-posterior, introduciendo el NFE a través de una fosa nasal, pasando a través del meato inferior y piso nasal, lo que permite realizar una inspección del paladar duro y blando, además de la visualización directa del esfínter velofaríngeo, de sus estructuras y movimientos realizados durante el habla y la deglución en forma dinámica; después se 
le realizó a cada paciente una evaluación fonológica pidiéndole que formulará fonemas velares $\mathrm{k} / \mathrm{c} / \mathrm{q}$ tales como: «Kuki quiere coco», «Kike come queso»y "Cuca» con base en los criterios de Henningsson, ${ }^{13}$ obteniendo una imagen del patrón velofaríngeo que se produjo en el momento de la fonación.

\section{RESULTADOS}

Análisis de datos: fueron colectados, tabulados y analizados en un ordenador, con paquete estadístico STATA ${ }^{\circledR}$ MP versión 14 , se utilizó análisis estadístico de $\chi^{2}$ con una $p<0.05$.

Se registraron 118 pacientes con paladar hendido que fueron tratados quirúrgicamente en el Hospital Pediátrico de Peralvillo, en el periodo de enero de 2017 a febrero de 2020, sólo 16 cumplieron con los criterios de inclusión, ${ }^{11}$ correspondieron al género masculino $(68.75 \%)$, cinco al femenino (31.25\% SE 1.2, DE 5.08, IC 95\% 6.47-11.89). El promedio de edad del sexo masculino fue de 9.8 (DE 5.7) y del sexo femenino de 7.8 (DE 3.1).

De forma ascendente a descendente los diagnósticos fueron: labio y paladar hendido unilateral izquierdo-LPHUI (43.75\% DE 6.9), seguido del paladar hendido submucoso-PHS (37.5\% DE 3.7), labio y paladar hendido bilateral-BI (12.5\% DE 2.1) y labio y paladar hendido unilateral derecho (6.25\%).

La esfinteroplastia fue el principal procedimiento en el velo del paladar en comparación con la veloplastia doble zeta (Figura 2); el promedio de edad de los pacientes sometidos a esta técnica quirúrgica fue de 7.8 (DE 5.1) y 11.5 (DE 4.3) años, respectivamente.

Técnica quirúrgica (velo del paladar)

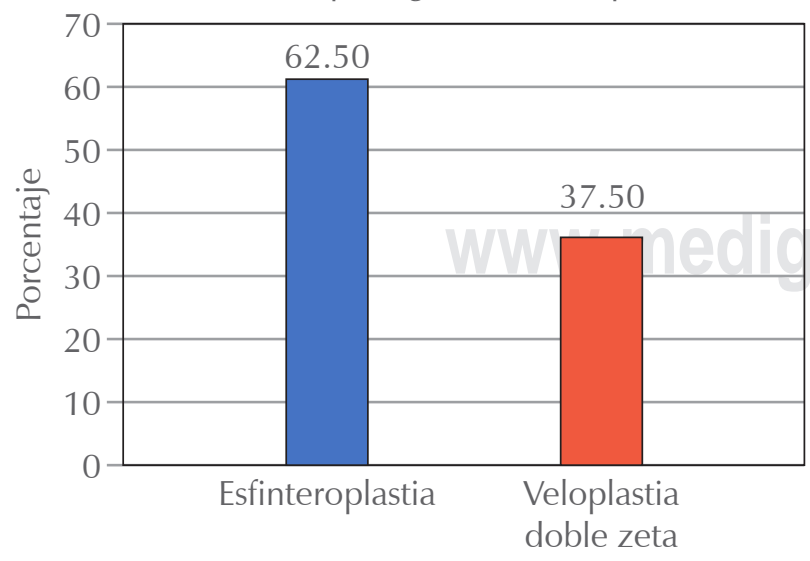

Figura 2: Muestra la técnica quirúrgica empleada para cierre de velo de paladar.

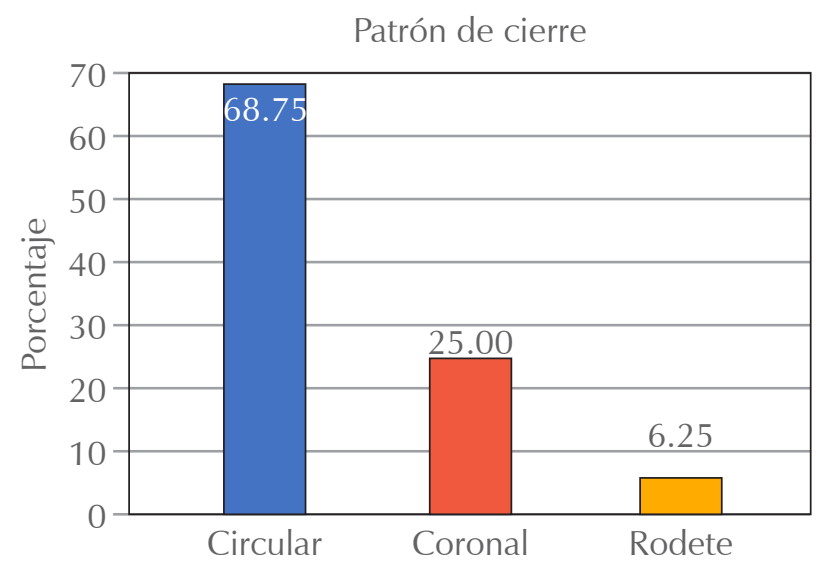

Figura 3: Patrones de cierre velofaríngeo que se presentaron en este estudio.

La técnica quirúrgica principalmente utilizada fue la esfinteroplastia lineal de Bardach 62.5\% (SE 1.6, DE 5.18, IC 95\% 4.09-11.50) y la doble z Furlow se utilizó en seis ocasiones (37.5\% SE 1.7, DE 4.37, IC 95\% 6.47-11.50); se requirió de cirugías agregadas, $43.75 \%$ Bardach, veau Wardill Kilner $25 \%$, von Lagenback $6.25 \%$ y ninguna $25 \%$.

El patrón de cierre circular fue el más prevalente (media 10.1 años, DE 5.3) realizada en ocho masculinos y tres femeninos, seguido del coronal (media 5.2 años, DE 1.7) ejecutada en cuatro niños, dos femeninos y dos masculinos y rodete de Passavant (media 14 años) efectuada en un masculino (Figura 3) $\mathrm{p}<0.05$.

\section{DISCUSIÓN}

En la presente investigación se determinaron los patrones más comunes de cierre velofaríngeo descritos en la literatura, en pacientes con paladar hendido, posterior al cierre quirúrgico mediante las técnicas doble z-plastia de Furlow y la esfinteroplastia lineal de Bardach.

La esfinteroplastia lineal de Bardach es una variante a la técnica de Wardill, en la cual se realiza una incisión en el margen de la fisura, prolongando las incisiones por detrás del plano de la úvula hacia posterior, siguiendo los pilares faríngeos posteriores de la amígdala en sus dos tercios superiores, se realiza una disección roma con la finalidad de movilizar el músculo palatofaríngeo, proporcionando con esto un doble objetivo de dinámica velar, estrechando la proximidad de las paredes faríngeas laterales, realizándolo de la misma manera de ambos lados, 
suturando después los colgajos en tres planos: nasal, muscular y oral respectivamente, a nivel de los pilares posteriores y obteniendo una elongación lineal velar de 15 a 20 milímetros por detrás de la posición de la úvula, lo que se ve reflejado en una mayor longitud y dinámica del movimiento del velo al involucrar el pilar posterior, limitando la invasión a la pared faríngea posterior y eliminando morbilidades asociadas a la técnica original (Figura 4). 11,12,19,21

Las técnicas quirúrgicas realizadas en este estudio, como la esfinteroplastia lineal y la doble z-plastia son efectivas para lograr un patrón de cierre fisiológicamente adecuado, siendo la z-plastia de Furlow más efectiva para acercarse a éste, con una taza de éxito de $96.6 \%$, según Wong y colaboradores, ${ }^{14}$ quienes refieren que sus pacientes no requirieron de un segundo acto quirúrgico por presencia de insuficiencia velofaríngea.

El cierre velofaríngeo es acompañado de la contracción de diferentes músculos incluyendo al elevador del velo palatino, músculo de la úvula,
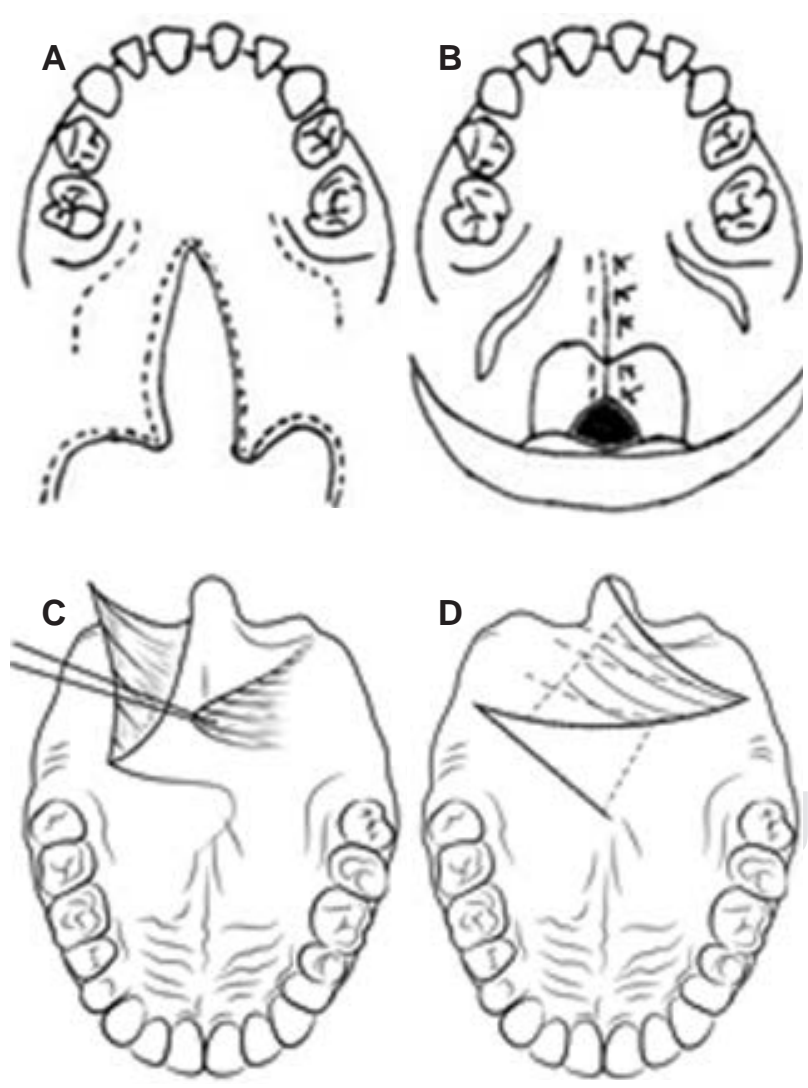

Figura 4: A-B) Técnica de Bardach. C-D) Técnica doble z-plastia de Furlow.

Tomado y modificado de: Michael S et al, ${ }^{20}$ Monserat $\mathrm{E}$ et al. ${ }^{21}$

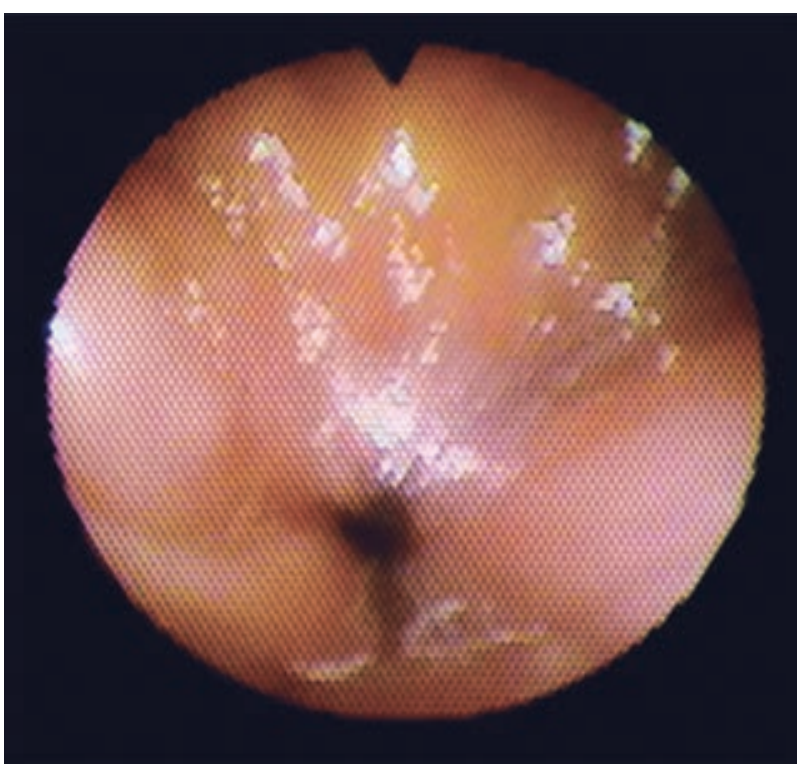

Figura 5: Patrón de cierre velofaríngeo circular.

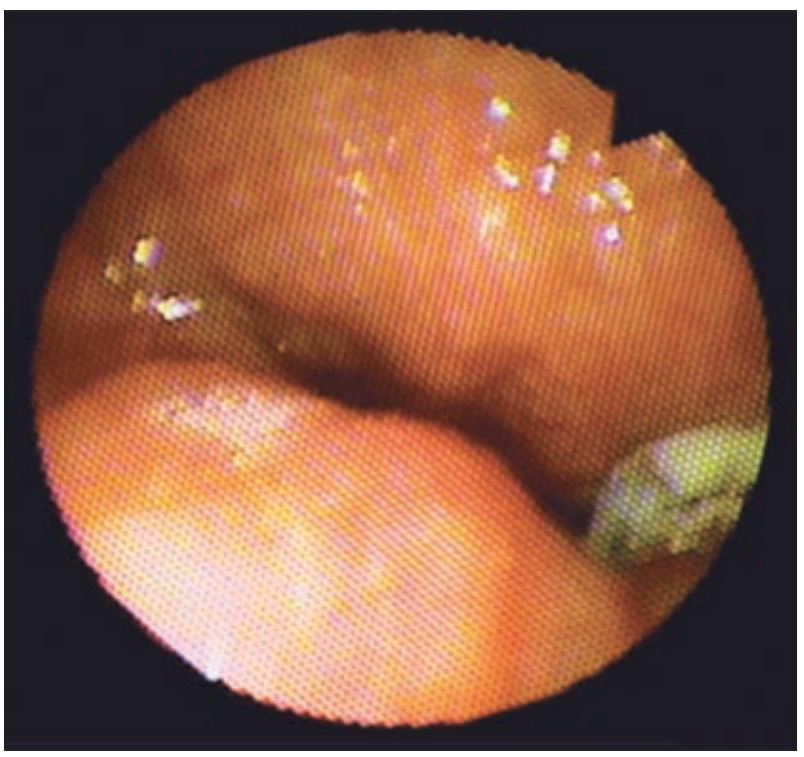

Figura 6: Patrón de cierre velofaríngeo coronal.

constrictor superior de la faringe, palatofaríngeo, palatogloso, salpingofaríngeo y el tensor del velo del paladar que es responsable de la función de la trompa de Eustaquio.

Los patrones de cierre velofaríngeo representan una descripción de una condición estática del esfínter en la fase terminal del cierre, por lo que el uso de nasofibroendoscopia es aceptado como el mejor método de análisis clínico para observar el esfínter 
velofaríngeo. Golding-Kushner y colaboradores, ${ }^{8}$ describieron a la nasofibroendoscopia como sistema de cuantificación y descripción de los movimientos de las estructuras del esfínter velofaríngeo, tal como se realizó en nuestro estudio obteniendo los ángulos e imágenes clínicas adecuadas para observar dicho esfínter (Figuras 5 y 6).

El patrón de cierre velofaríngeo dominante en la población mundial sin fisura palatina es el circular, los patrones de cierre velofaríngeo son categorizados de acuerdo a la pared que contribuye principalmente al cierre del esfínter velofaríngeo durante el habla. El cierre de la válvula velofaríngea es un mecanismo tridimensional que envuelve la función del velo y las paredes faríngeas, por lo que el patrón coronal ocurre cuando hay un movimiento excesivo del velo del paladar, que se desplaza a la pared faríngea posterior con menor movimiento de otras estructuras. El patrón sagital corresponde al movimiento de las paredes faríngeas laterales hacia la línea media con menor movimiento de la pared faríngea posterior y el velo del paladar. El patrón circular cuando todas las estructuras se desplazan juntas hacia la línea media y finalmente el patrón circular con rodete de Passavant, en el cual esta estructura que está formada por fibras del músculo constrictor superior de la faringe contribuye al cierre en la línea media (Figura 1). 3,5,7,8,17,20

En nuestro estudio, los resultados coinciden con los hallazgos descritos por MacKenzie y su grupo, ${ }^{15}$ en los cuales reportaron que el patrón de cierre velofaríngeo que se presentó con más frecuencia fue el Circular en un 58\%, igualmente en la investigación de Madrid y colaboradores ${ }^{6}$ reportaron la prevalencia del patrón circular en un 39.02\%.

En este estudio se confirmó la presencia de tres de los cuatro patrones descritos por Skolnick y Croft, ${ }^{5}$ tal como lo observaron Prada Madrid y su equipo, ${ }^{6}$ quienes concluyen que el único patrón no registrado fue el sagital, asociándolo a que en este patrón se encuentra prácticamente inactivo el músculo elevador del paladar, no requirieron de un segundo acto quirúrgico por presencia de insuficiencia velofaríngea.

\section{CONCLUSIONES}

El adecuado lenguaje depende de la integridad y funcionalidad de las estructuras velofaríngeas, el objetivo de una intervención quirúrgica en pacientes con paladar hendido es restaurar dicha anatomía para obtener una adecuada función velofaríngea.
Pocos estudios describen los patrones de cierre velofaríngeo, así como la frecuencia de presentación de los patrones de cierre en pacientes con paladar hendido, pero concuerdan en que fisiológicamente el patrón circular es el más común en el mundo.

Es importante mencionar que en este estudio las técnicas que se utilizaron ayudan más al desarrollo fisiológico de un patrón circular adecuado, ya que proveen un alargamiento adecuado del paladar sin la necesidad de agregar otros procedimientos quirúrgicos, además de que previenen la contractura longitudinal de la cicatriz, ayudando así a mejorar el lenguaje, la deglución y la ventilación del oído medio.

Esta investigación puede ser utilizada para seguir realizando otras en las cuales se tenga una mayor muestra para el análisis de patrones de cierre velofaríngeo y se comparen técnicas quirúrgicas para saber con cuál de ellas se obtiene el patrón más adecuado para la fonación en casos de habla hispana, así como otros estudios en los cuales se valore la funcionalidad de técnicas quirúrgicas de cierre de hendiduras palatinas mediante la observación de la función del músculo tensor del velo del paladar que ayuda al cierre de la trompa de Eustaquio.

\section{AGRADECIMIENTOS}

Al Hospital Pediátrico de Peralvillo por las facilidades otorgadas para este estudio, en especial al Dr. Hernán Castilla Canseco Jefe de la Clínica de Labio y Paladar Hendido de dicho hospital y a todos los doctores involucrados en este trabajo.

\section{REFERENCIAS}

1. Beer AJ, Hellerhoff P, Zimmermann A, Maddy K, Sader R, Rummeny EJ et al. Dynamic Near -Real- Time magnetic resonance imagin for analysing the velopharingeal closure in comparison with videofluoroscopy. Journal of Magnetic Resonance Imaging. 2004; 20: 791-797.

2. Sadove AM, van Aalst JA, Culp JA. Cleft palate repair: art and issues. Clin Plast Surg. 2004; 31: 231-241.

3. Perry JL. Anatomy and physiology of the velopharyngeal mechanism. Semi Speech Lang. 2011; 32: 83-92.

4. Naiman N, Disant F. Cirugía de la Insuficiencia velofaríngea. EMC Consulte. 2001; E-46-310.

5. Skolnick M, McCall G, Carnes M. The sphincteric macanism of velopharyngeal closure. New York, NY: McGraw-Hill; 2009, 653.

6. Prada MJR, García VT, Echeverri B, MP, Tavera HMC. Patterns of velopharyngeal closure: comparative study between healthy population and patients with cleft palate. Cir Plást Iberotatinoam. 2010; 36: 305-312.

7. Fisher D, Sommerlad B. Cleft lip, cleft palate, and velopharyngeal insufficiency. CME. Plast Reconstr Surg. 2011; 128 (4): 342e-360e.

8. Golding-Kushner KJ, Argamaso RV, Cotton RT. Standardization for the reporting of nasopharyngoscopy and multiview videofloroscopy. 
A report from an international working group. Cleft Palate J. 1990; 27: 337-347.

9. Engelke W, Poppelreuter S, Burns T, Quantitative Analysis of the velopharingeal Sphincter function during speech. Cleft Palate Craniofac J. 2000; 37 (2): 157-165.

10. Hopper RA, Tse R, Smartt J, Swanson J, Kinter S. Cleft palate repair and velopharyngeal dysfunction. Plastic Reconstr Surg. 2014; 133 (6): 852-864.

11. Paliobei V, Psifidis A, Anagnostopoulos D. Hearing and speech assessment of cleft palate patients after palatal closure. Long term results. Int J Pediatr Otorhinolaryngol. 2005; 69: 1373-1381.

12. Smith M, Losse J. Cleft palate repair. Clin Plastic Surg. 2014; 41: 189-2010.

13. Arboleda C, Dueñas B. Frases en español para distintos fonemas. CCC-SLP. Leadersproject. 2009

14. Wong LS, Lim E, Lu TC, Chen PKT. Management of velopharyngeal insufficiency by modified Furlow palatoplasty with pharyngeal flap: a retrospective outcome review. Int J Oral Maxillofac Surg. 2019; 48: 703-707.

15. MacKenzie-Stepner K, Witzel MA, Stringer DA, Laskin R. Velopharyngeal insuficiency due to hypertrophic tonsils. A reporte of two cases. Int J Pediatr Otorhinolaryngol. 1987; 14: 57-63.
16. Pearson G, Kirschner R. Surgery for clerf palate and velopharyngeal dysfunction. Seminars in Speech and Language. 2011; 32 (2): 179-190.

17. Riski JE, Ruff GL, Georgiade GS, Barwick WJ, Edward PD. Evaluation of the sphincter pharyngoplasty. Cleft Palate Craniofac J. 1992; 29: 254-261.

18. Witt PD, Marsh JL, Arlis H, Grames LM, Ellis RA, Pilgrim TK. Quantification of Dynamic velopharyngeal port excursion following sphincter pharyngoplasty. Plast Reconstr Surg. 1998; 101: 1205-1211.

19. Campbell A, Costello BJ, Ruiz RL. Cleft lip and palate surgery: an update of clinical outcomes for primary repair. Oral Maxillofacial Surg Clin N Am. 2010; 22: 43-58.

20. Michael S, Arun K. Surgical management of velopharyngeal insufficiency. Clin Plastic Surg. 2014; 41: 253-270.

21. Monserat $\mathrm{E}$, Sillet $\mathrm{M}$. Paladar hendido tratamiento quirúrgico. Acta Odontológica Venezolana. 2002; 40 (3): 289-293.

22. Ragab A. Cerclage sphincter pharyngoplasty: a new technique for velopharyngeal insufficiency. Int J Pediatr Otorhinolaryngol. 2007; 71: 793-800.

23. Rudnick EF, Sie KC. Velopharyngeal insufficiency: current concepts in fiagnosis an management. Curr Opin Otolaryngol Head Neck Surg. 2008; 16: 530-535. 